\title{
An evaluation of factors affecting adherence to antiepileptic drugs in patients with epilepsy: a cross-sectional study
}

Ranjana Gurumurthy ${ }^{1}$, MSc, PhD, Kulkarni $\underline{\text { Chanda }}^{2}$, MD, PhD, GRK $\underline{\text { Sarma }}^{3}$, MD, DM

INTRODUCTION Adherence to antiepileptic drug (AED) therapy is important for controlling seizures in patients with epilepsy (PWE). It is vital to identify the factors influencing adherence to AED therapy using validated tools. This study aimed to evaluate the pattern and extent of AED adherence among PWE and to identify the factors that influence adherence. METHODS This was a cross-sectional study involving PWE who had a confirmed diagnosis. Treatment adherence was assessed using the four-item Morisky Medication Adherence Scale. Univariate analysis with chi-square test was used to observe the association between different variables and AED adherence. Binary logistic regression analysis was used to identify the predictors of adherence.

RESULTS 451 PWE (mean age $27.3 \pm 8.1$ years) were enrolled in the study; 251 (55.7\%) were male and 198 (43.9\%) were from the lower socioeconomic class. 326 (72.3\%) patients had high adherence to AED therapy, while 125 (27.7\%) had low adherence. AED adherence was significantly associated with socioeconomic status $(p=0.043)$ and type of epilepsy $(p=0.033)$. However, no significant difference was observed between adherence and age, gender, marital status, epilepsy duration, number and type of AEDs, and occurrence of adverse drug reactions. Patients with focal epilepsy and those from the middle/lower-middle socioeconomic classes were less likely to be nonadherent. The primary reason for nonadherence was forgetfulness.

CONCLUSION This study found that a majority of PWE have optimal rates of AED adherence and that forgetfulness is the primary reason for nonadherence among PWE.

Keywords: antiepileptic drugs, epilepsy, Morisky Medication Adherence Scale, treatment adherence

\section{INTRODUCTION}

Epilepsy affects 70 million people worldwide.(1) Long-term antiepileptic drug (AED) administration remains the mainstay of epilepsy treatment. In up to $67 \%$ of patients with epilepsy (PWE), AEDs effectively eliminate or reduce the frequency of seizures. ${ }^{(2)}$ Medication adherence refers to the extent to which a patient's behaviour corresponds with the recommendations of a health professional. Medication adherence is usually better when there is greater patient involvement in the treatment choice, as well as cooperation and mutual agreement between the health provider and the patient. ${ }^{(2)}$ Nonadherence rates among PWE are reported to be $30 \%-50 \% .{ }^{(3)}$ It is well established that patients with suboptimal adherence levels are more likely to have seizures that are associated with increased number of hospital admissions and healthcare costs. ${ }^{(4)}$ Factors influencing adherence to AEDs have been described; these include patient-, treatment- and health system-related factors. ${ }^{(5)}$ The promotion of medication adherence is considered to be an important component of pharmaceutical care practice. Several methods have been used to measure therapeutic adherence, including self-report, pill count, appointment attendance, medication refill history, blood or urine drug levels and drug diary. ${ }^{(6)}$ Indirect methods, such as self-reports and patient interviews, are the simplest and most common methods used for measuring medication adherence. ${ }^{(7,8)}$
To date, most of the studies conducted to evaluate AED adherence among PWE did not account for the influence of a wide range of variables (e.g. demographic, disease and treatment patterns). There are not many studies in the Indian literature that examine the factors that influence AED adherence. Identifying the factors associated with AED adherence would allow the development of strategies to improve adherence. Thus, the present study aimed to evaluate the pattern and extent of AED adherence among PWE and to identify the factors that influence adherence.

\section{METHODS}

This cross-sectional study was conducted by the Division of Clinical Pharmacology at the outpatient and inpatient clinics of the Department of Neurology, St John's Medical College and Hospital, Bangalore, India, over a period of 1.5 years (January 2012 to July 2013). Ethical approval was obtained from the institution's Ethical Review Board. Patients aged $\geq 18$ years who were receiving AED treatment were eligible for inclusion in the study. Only those who gave their consent for participation were enrolled. Pregnant or lactating women and patients with comorbidities were excluded from the study.

Data was collected using a specially designed case record form that included items on demographic/family history, seizure history, duration of epilepsy, AEDs prescribed and occurrence of

${ }^{1}$ Department of Pharmacology, St John's Medical College and Hospital, ${ }^{2}$ Department of Pharmacology, Sri Rajarajeshwari Medical College and Hospital, ${ }^{3}$ Department of Neurology, St John's Medical College and Hospital, Bangalore, India

Correspondence: Dr Chanda Kulkarni, Research Consultant and Professor of Pharmacology, Central Research Lab, Department of Pharmacology, Sri Rajarajeshwari Medical College and Hospital, 202 Mysore Road, Kambipura, Bengaluru, Karnataka 560074, India. drchandakulkarni@gmail.com 
adverse drug reactions (ADRs). The participants were classified according to their age, gender, marital status and socioeconomic class. To determine the socioeconomic class of the participants, Kuppuswamy's socioeconomic status scale was used; the scale takes into consideration the education, occupation and family income of the participants. ${ }^{(9)}$ Epilepsy was diagnosed based on detailed medical history, neurological evaluation by a qualified neurologist, and electroencephalography and neuroimaging techniques (i.e. computed tomography and magnetic resonance imaging). Details on the patients' AED treatment, including the generic name of the AED, dose and observed ADRs, were also collected.

Adherence to AED treatment was assessed using the four-item Morisky Medication Adherence Scale (MMAS-4), ${ }^{(10)}$ a standardised, validated questionnaire for measuring self-reported medication adherence. The four items of the MMAS- 4 assess whether: (a) the patient has ever forgotten to take medication; (b) the patient has ever had problems remembering to take medication; (c) the patient has stopped medication due to worsening symptoms; and (d) the patient has stopped medication due to alleviating symptoms. ${ }^{(11)}$ Each item is scored as either 0 (Yes) or 1 (No). The score of each item is then summed to give a range of scores from 0 to 4 . A score of 3-4 suggests that the patient is adherent, while a score of $\leq 2$ suggests that the patient is nonadherent.

Data was expressed as frequency (percentage). Descriptive statistical analysis was used to analyse the data, and univariate analysis with chi-square test was used to observe the association between the variables and adherence. Binary logistic regression analysis was used to identify the predicting factors of adherence to treatment. A p-value $<0.05$ was considered statistically significant.

\section{RESULTS}

A total of 451 PWE were enrolled in the study. 251 (55.7\%) patients were male and the majority $(n=318,70.5 \%)$ were aged 18-30 years (Table I). The mean age of the participants was 27.3 \pm 8.1 years. More than half of the PWE $(n=237,52.5 \%)$ were unemployed; of these, 114 (48.1\%) were housewives, 98 (41.4\%) were students and 25 (10.5\%) were unable to find employment due to their epilepsy. Most of the PWE ( $n=198,43.9 \%$ ) belonged to the lower/upper-lower socioeconomic class.

Focal epilepsy was more common in our study cohort $(\mathrm{n}=298,66.1 \%)$ than generalised epilepsy $(\mathrm{n}=153,33.9 \%)$ (Table II). About half of the participants $(n=228,50.6 \%)$ had $1-3$ episodes of seizure per year. $104(23.1 \%)$ patients were on old AEDs (e.g. phenytoin, valproic acid), 220 (48.8\%) were on new AEDs (e.g. oxcarbazepine, levetiracetam) and 127 (28.2\%) were on combination AED therapy. More than half of the PWE $(n=241$, $53.4 \%$ ) were on AED monotherapy, while 210 (46.6\%) were on AED polytherapy. Among those who were on polytherapy, $160(76.2 \%)$ were on two types of AEDs, 46 (21.9\%) were on three types of AEDs, and 4 (1.9\%) were on more than three types of AEDs.

Among the 241 PWE who were prescribed AED monotherapy, the most frequently prescribed old AED was phenytoin $(\mathrm{n}=34,14.1 \%)$, followed by valproic acid $(\mathrm{n}=30,12.4 \%)$. The
Table I. Demographic characteristics of the patients with epilepsy $(n=451)$.

\begin{tabular}{|c|c|}
\hline Variable & No. (\%) \\
\hline \multicolumn{2}{|l|}{ Age group (yr) } \\
\hline $18-30$ & $318(70.5)$ \\
\hline $31-49$ & $133(29.5)$ \\
\hline \multicolumn{2}{|l|}{ Gender } \\
\hline Male & $251(55.7)$ \\
\hline Female & $200(44.3)$ \\
\hline \multicolumn{2}{|l|}{ Marital status } \\
\hline Married & $236(52.3)$ \\
\hline Unmarried & $215(47.7)$ \\
\hline \multicolumn{2}{|l|}{ Highest educational level } \\
\hline Professional or honours & $21(4.7)$ \\
\hline Graduate or postgraduate & $108(23.9)$ \\
\hline Intermediate or high school diploma & $101(22.4)$ \\
\hline High school certificate & $157(34.8)$ \\
\hline Middle school certificate & $36(8.0)$ \\
\hline Primary school certificate & $8(1.8)$ \\
\hline Illiterate & $20(4.4)$ \\
\hline \multicolumn{2}{|l|}{ Occupation } \\
\hline Professional & $14(3.1)$ \\
\hline Semi-professional & $16(3.5)$ \\
\hline Clerk, shop owner or farmer & $29(6.4)$ \\
\hline Skilled worker & $150(33.3)$ \\
\hline Semiskilled worker & $2(0.4)$ \\
\hline Unskilled worker & $3(0.7)$ \\
\hline Unemployed & $237(52.5)$ \\
\hline \multicolumn{2}{|l|}{ Monthly income (INR) } \\
\hline$\geq 32,050$ & $34(7.5)$ \\
\hline $16,020-32,049$ & $57(12.6)$ \\
\hline $12,020-16,019$ & $48(10.6)$ \\
\hline $8,010-12,019$ & $97(21.5)$ \\
\hline $4,810-8,009$ & $144(31.9)$ \\
\hline $1,601-4,809$ & $56(12.4)$ \\
\hline$\leq 1,600$ & $15(3.3)$ \\
\hline \multicolumn{2}{|l|}{ Socioeconomic class* } \\
\hline Upper & $11(2.4)$ \\
\hline Upper-middle & $89(19.7)$ \\
\hline Middle/lower-middle & 153 (33.9) \\
\hline Lower/upper-lower & $198(43.9)$ \\
\hline
\end{tabular}

*Determined using Kuppuswamy's socioeconomic status scale. ${ }^{(9)}$ INR: Indian rupee

new AEDs used in AED monotherapy included oxcarbazepine $(\mathrm{n}=66,27.4 \%)$, levetiracetam $(\mathrm{n}=53,22.0 \%)$ and topiramate $(\mathrm{n}=6,2.5 \%)$. The most common AED dual therapy was oxcarbazepine with clobazam $(\mathrm{n}=31,19.4 \%)$, and the most frequently used add-on AED was clobazam (58.2\%). Patients with focal epilepsy received phenytoin, carbamazepine, oxcarbazepine and/or levetiracetam, while those with generalised epilepsy received valproic acid, levetiracetam and/or lamotrigine.

Among the 451 PWE, 222 (49.2\%) reported having ADRs; of these, 175 (78.8\%) had ADRs involving the central nervous system (e.g. impaired memory, tiredness, drowsiness, headache 
Table II. Clinical characteristics of the patients with epilepsy $(n=451)$.

\begin{tabular}{|c|c|}
\hline Variable & No. (\%) \\
\hline \multicolumn{2}{|l|}{ Duration of epilepsy (yr) } \\
\hline$\geq 1$ & $366(81.2)$ \\
\hline$<1$ & $85(18.8)$ \\
\hline \multicolumn{2}{|l|}{ Type of epilepsy } \\
\hline Focal & $298(66.1)$ \\
\hline Generalised & $153(33.9)$ \\
\hline \multicolumn{2}{|l|}{ Type of AED therapy } \\
\hline Monotherapy & $241(53.4)$ \\
\hline Polytherapy & $210(46.6)$ \\
\hline \multicolumn{2}{|l|}{ Type of AED used } \\
\hline Old & $104(23.1)$ \\
\hline New & $220(48.8)$ \\
\hline Combination & $127(28.2)$ \\
\hline \multicolumn{2}{|l|}{ Seizure frequency } \\
\hline $1-3$ per yr & $228(50.6)$ \\
\hline$\geq 1$ per mth & $21(4.7)$ \\
\hline Seizure-free for $\leq 2 \mathrm{yr}$ & $59(13.1)$ \\
\hline Seizure-free for $>2 \mathrm{yr}$ & $60(13.3)$ \\
\hline Not applicable* & $83(18.4)$ \\
\hline \multicolumn{2}{|l|}{ Adverse drug reactions } \\
\hline Yes & $222(49.2)$ \\
\hline No & $229(50.8)$ \\
\hline
\end{tabular}

*Not applicable as patients were newly diagnosed. AED: antiepileptic drug

and depression), 15 (6.8\%) had aphthous ulcers, $10(4.5 \%)$ experienced weight gain, 7 (3.2\%) had gastritis, $6(2.7 \%)$ had bone pain, $5(2.3 \%)$ had rashes and $4(1.8 \%)$ had pimples.

A small proportion of the PWE (27.7\%) answered "Yes" (i.e. indicated nonadherence to AED therapy) to at least one of the four items in the MMAS-4 questionnaire. Forgetfulness was the most common reason for nonadherence $(n=91,32.5 \%)$ followed by having problems remembering $(n=82,29.3 \%)$ and stopping medication when feeling worse $(\mathrm{n}=56,20.0 \%)$ or when feeling better $(n=51,18.2 \%$ ) (Fig. 1). The average score of the patients on MMAS-4 was $3.37 \pm 1.06$, which is close to the 'very good' rating.

Based on the results of MMAS-4, $326(72.3 \%)$ of the PWE were adherent and $125(27.7 \%)$ were nonadherent to AED therapy. Socioeconomic status and type of epilepsy were found to be significantly associated with adherence $(p=0.043$ and $p=0.033$, respectively), while age, gender, marital status, duration of epilepsy, type of AED therapy, type of AED used and the occurrence of ADRs were not found to be significantly associated with adherence (Table III).

Factors that were found to be significantly associated with medication adherence in the univariate analysis (i.e. socioeconomic class $[p=0.043$ ] and type of epilepsy $[p=0.033]$ ) were included in the multiple regression analysis. The results of the multiple logistic regression analysis are summarised in Table IV. Logistic regression analysis indicated that PWE who had focal epilepsy were less likely to be nonadherent than PWE who had generalised epilepsy (odds ratio [OR] 0.620, 95\% confidence interval $[\mathrm{Cl}]$ 0.403-0.954) and that PWE who

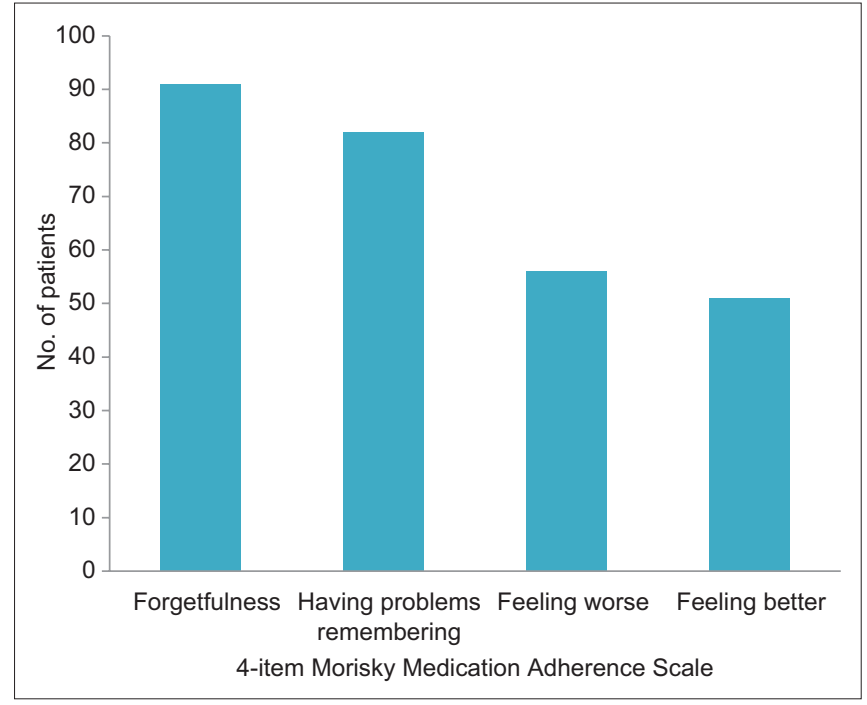

Fig. 1 Bar graph shows the reasons for nonadherence to antiepileptic drug therapy among patients with epilepsy.

belonged to the middle/lower-middle socioeconomic class were less likely to be nonadherent than PWE from the lower/upperlower socioeconomic class (OR 0.517, 95\% Cl 0.317-0.841).

\section{DISCUSSION}

Adherence to AED is a challenge in the management of epilepsy. Although poor adherence is considered to be one of the major causes of non-responsiveness to AED therapy, this has not been studied extensively in India. Hence, we assessed the extent of AED adherence, as well as the factors influencing AED adherence among PWE.

Few studies have used self-reporting measures of adherence to assess adherence among PWE. ${ }^{(12,13)}$ MMAS-4 was used in the present study and it showed that the majority (72.3\%) of PWE in our study cohort adhered to the prescribed regimen. Another study that used a self-reporting measure of adherence reported a $51.9 \%$ adherence rate. ${ }^{(13)}$ These results suggest that the PWE in our study were more adherent to their prescribed medication than patients in other studies that assessed adherence using selfreporting measures. Differences in the rate of adherence may be due to the unique characteristics of the study populations (e.g. differences in the patient attitude toward adherence due to cultural or educational influences, or clinicians' approach) and differences in the criteria used to measure adherence. ${ }^{(14)}$

In the present study, we did not find any significant differences in the rate of nonadherence among the age groups and between the genders. This finding is similar to that of Liu et al's study, ${ }^{(13)}$ which did not find any demographic differences (i.e. gender and age) between AED-adherent and AED-nonadherent patients. A study by Johnbull et $\mathrm{al}^{(15)}$ reported a similar observation, in that gender did not affect the adherence rate in their study.

We found a significant association between AED adherence and lower/upper-lower socioeconomic class. This is in contrast to the findings of Asadi-Pooya, ${ }^{(12)}$ who found no significant association between AED adherence and socioeconomic status (measured based on parental smoking, place of residence and educational level). In a study conducted by Das et al in India, ${ }^{(16)}$ 
Table III. Results of univariate analysis comparing patients with epilepsy who were adherent and nonadherent to medication ( $\mathrm{n}=451$ ).

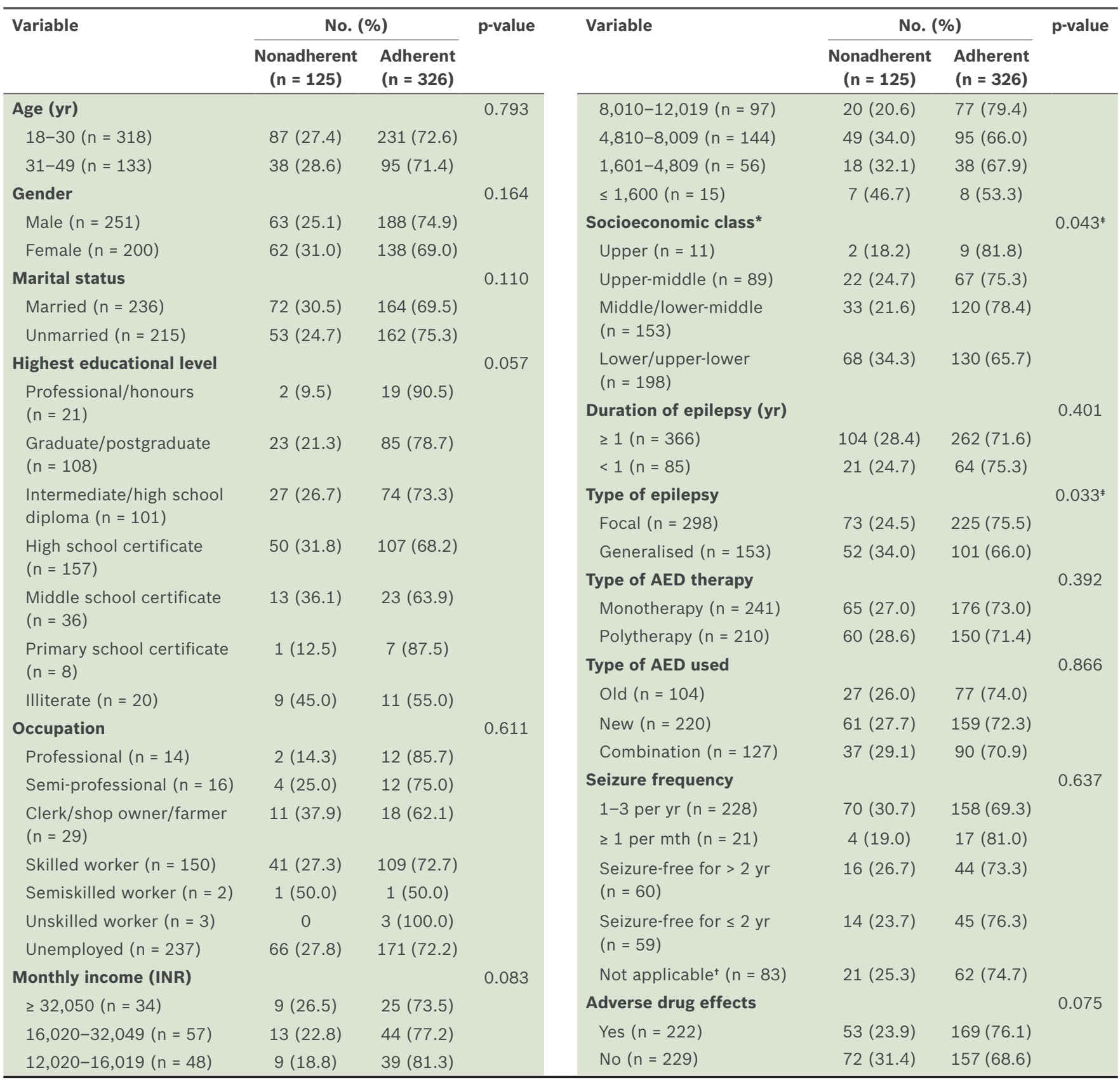

*Determined using Kuppuswamy’s socioeconomic status scale.(9) †Not applicable as patients were newly diagnosed. $¥$ Statistically significant at $p<0.05$. AED: antiepileptic drug; INR: Indian rupee

Table IV. Results of the logistic regression analysis evaluating the factors associated with nonadherence.

\begin{tabular}{lcccc}
\hline Variable & B & p-value & OR & $95 \% \mathbf{C l}$ \\
\hline Socioeconomic class & & & & \\
$\quad$ Upper & -0.878 & 0.416 & 0.087 & $0.087-1.993$ \\
$\quad$ Upper-middle & -0.453 & 0.117 & 0.636 & $0.317-0.841$ \\
$\quad$ Middle/lower-middle & -0.660 & 0.008 & 0.517 & $0.317-0.841$ \\
Type of epilepsy & & & & \\
$\quad$ Focal epilepsy & -0.478 & 0.030 & 0.620 & $0.403-0.954$ \\
\hline
\end{tabular}

$\mathrm{Cl}$ : confidence interval; OR: odds ratio

the following socioeconomic factors were found to be related to discontinuation of AED treatment: unemployment; loss of job; marital disharmony; spiritual illusions about epilepsy; and lack of availability of medications in local areas. The findings of the present study suggest that PWE who are of a lower socioeconomic class (based on Kuppuswamy's socioeconomic status scale, which takes into account education, occupation and income $)^{(9)}$ may be at a higher risk of nonadherence.

In the present study, the duration of epilepsy did not have a significant association with adherence among the PWE. This finding is similar to the results reported by Gabr and Shams, ${ }^{(17)}$ but differs from the results of Kyngäs' study, which showed that a duration of epilepsy $>3$ years was significantly related to adherence to medications. ${ }^{(18)}$ However, a significant association between generalised epilepsy and nonadherence to AED was found in the present study, as well as in the study by Samsonsen et $\mathrm{al}^{\left({ }^{(19)}\right.}$ the reason for this observation is elusive. One possible explanation that may account for the higher rate of adherence is 
that episodes of focal epilepsy are more frequent than generalised epilepsy. However, Liu et al ${ }^{(13)}$ did not find any relationship between the type of epilepsy and adherence. In terms of frequency, we did not find any significant association between frequency of seizure and adherence in our study, unlike Gabr and Shams, ${ }^{(17)}$ who found that seizure frequency was higher among PWE who were nonadherent.

Our findings regarding the influence of AED therapy type on the extent of adherence are in agreement with those of Sweileh et al. (20) Both studies showed no significant difference in medication adherence between patients on monotherapy and those on polytherapy. In contrast, the studies by Gabr and Shams, ${ }^{(17)}$ Bautista and Rundle-Gonzalez ${ }^{(21)}$ and Harimanana et $\mathrm{al}^{(22)}$ found that patients on monotherapy were significantly more adherent to medication than those who were on polytherapy. This has been attributed to the complexity of a treatment regimen ${ }^{(7)}$ involving a larger number of pills that need to be taken at different time intervals, which increases the likelihood of missed doses. ${ }^{(23)}$ In the present study, we did not find any significant relationship between the occurrence of ADRs and adherence, even though ADRs that are related to the central nervous system may increase the likelihood of nonadherence. Our finding differs from that of Martins et al's study, ${ }^{(6)}$ which found a statistically significant association between the occurrence of ADRs and AED adherence among their cohort, which consisted mainly of patients suffering from juvenile myoclonic epilepsy.

Multiple logistic regression analysis of the patient demographics and clinical factors revealed that socioeconomic status and type of epilepsy were the variables that best explained nonadherence in our cohort. Other than that, the most common reason for nonadherence was forgetfulness of PWE. This finding is in accordance with those of studies conducted by Liu et $\mathrm{al}^{(13)}$ and Paschal et $\mathrm{al}^{(24)}$ who reported that the primary reason for nonadherence in their study cohort was forgetfulness $(69.6 \%$ and $32.5 \%$, respectively). Johnbull et $\mathrm{al}^{(15)}$ observed that forgetfulness was the reason for nonadherence in only $40.7 \%$ of their study cohort.

MMAS-4, which is a validated and reliable tool with a significant linear relationship with adherence, was used to measure adherence in our study. However, this tool has the disadvantage of recall bias, and hence, has the tendency to overestimate adherence level. In order to reduce the bias related to the assessment of adherence using the four-item scale, it has been suggested that the responses given by PWE should be crossverified with data on prescription refills and/or the number of AEDs remaining with the patients, wherever possible.

To conclude, the present study found that socioeconomic class and type of epilepsy have a significant influence on the adherence of PWE to AED therapy, and that forgetfulness is the primary reason for AED nonadherence among PWE. The only limitation of the present study was that it was carried out in a tertiary healthcare setting, which made it difficult for us to extrapolate our findings to the general population. We suggest that further studies be conducted using multiple validated tools to measure adherence in a more varied population in order to better address the issue of AED adherence among PWE.

\section{REFERENCES}

1. Ngugi AK, Bottomley C, Kleinschmidt I, Sander JW, Newton CR. Estimation of the burden of active and life-time epilepsy: a meta-analytic approach. Epilepsia 2010; 51:883-90.

2. Elger CE, Schmidt D. Modern management of epilepsy: a practical approach. Epilepsy Behav 2008; 12:501-39.

3. Dilorio C, Shafer PO, Letz R, et al; Project EASE study group. Project EASE: a study to test a psychosocial model of epilepsy medication management. Epilepsy Behav 2004; 5:926-36.

4. Ettinger AB, Manjunath R, Candrilli SD, Davis KL. Prevalence and cost of nonadherence to antiepileptic drugs in elderly patients with epilepsy. Epilepsy Behav 2009; 14:324-9.

5. Rikir E, Grisar T, Sadzot B. [Treatment compliance in epileptic patients. A frequent and complex problem]. Rev Med Liege 2010; 65:366-9. French.

6. Martins HH, Alonso NB, Guilhoto LM, Guaranha MS, Yacubian EM. Adherence to treatment in patients with juvenile myoclonic epilepsy: correlation with quality of life and adverse effects of medication. J Epilepsy Clin Neurophysiol 2009; 15:192-6.

7. Shams ME, Barakat EA. Measuring the rate of therapeutic adherence among outpatients with T2DM in Egypt. Saudi Pharm J 2010; 18:225-32.

8. Girerd X, Hanon O, Anagnostopoulos K, et al. [Assessment of antihypertensive compliance using a self-administered questionnaire: development and use in a hypertension clinic]. Presse Med 2001; 30:1044-8. French.

9. Gururaj, Maheshwaran. Kuppuswamy's socio-economic status scale - A revision of income parameter for 2014. Int J Recent Trends Sci Tech 2014; $11: 1-2$.

10. Morisky DE, Green LW, Levine DM. Concurrent and predictive validity of a self-reported measure of medication adherence. Med Care 1986; 24:67-74.

11. Morisky DE, Ang A, Krousel-Wood M, Ward HJ. Predictive validity of a medication adherence measure in an outpatient setting. J Clin Hypertens (Greenwich) 2008; 10:348-54.

12. Asadi-Pooya AA. Drug compliance of children and adolescents with epilepsy. Seizure 2005; 14:393-5.

13. Liu J, Liu Z, Ding H, Yang X. Adherence to treatment and influencing factors in a sample of Chinese epilepsy patients. Epileptic Disord 2013; 15:289-94.

14. McAuley JW, McFadden LS, Elliott JO, Shneker BF. An evaluation of self-management behaviors and medication adherence in patients with epilepsy. Epilepsy Behav 2008; 13:637-41.

15. Johnbull OS, Farounbi B, Adeleye AO, Ogunrin O, Uche AP. Evaluation of factors influencing medication adherence in patients with epilepsy in rural communities of Kaduna State, Nigeria. Neurosci Med 2011; 2:299-305.

16. Das K, Banerjee M, Mondal GP, et al. Evaluation of socio-economic factors causing discontinuation of epilepsy treatment resulting in seizure recurrence: a study in an urban epilepsy clinic in India. Seizure 2007; 16:601-7.

17. Gabr WM, Shams ME. Adherence to medication among outpatient adolescents with epilepsy. Saudi Pharm J 2015; 23:33-40.

18. Kyngäs H. Compliance with health regimens of adolescents with epilepsy. Seizure 2000; 9:598-604.

19. Samsonsen C, Reimers A, Bråthen G, Helde G, Brodtkorb E. Nonadherence to treatment causing acute hospitalizations in people with epilepsy: an observational, prospective study. Epilepsia 2014; 55:e125-8.

20. Sweileh WM, Ihbesheh MS, Jarar IS, et al. Self-reported medication adherence and treatment satisfaction in patients with epilepsy. Epilepsy Behav 2011; 21:301-5.

21. Bautista RE, Rundle-Gonzalez V. Effects of antiepileptic drug characteristics on medication adherence. Epilepsy Behav 2012; 23:437-41.

22. Harimanana A, Clavel S, Chivorakul P, et al. Associated factors with adherence to antiepileptic drug in the capital city of Lao PDR. Epilepsy Res 2013; 104:158-66.

23. Jones RM, Butler JA, Thomas VA, Peveler RC, Prevett M. Adherence to treatment in patients with epilepsy: associations with seizure control and illness beliefs. Seizure 2006; 15:504-8.

24. Paschal AM, Rush, SE, Sadler T. Factors associated with medication adherence in patients with epilepsy and recommendations for improvement. Epilepsy Behav 2014; 31:346-50. 